\title{
A 5-component model for salt-induced hypertension
}

\author{
Violeta I. McLoone* John V. Ringwood* Bruce Van Vliet ${ }^{* *}$ \\ * Department of Electronic Engineering, National University of Ireland \\ Maynooth, Maynooth, Co. Kildare, Ireland (e-mail: \\ violeta.mcloone@eeng.nuim.ie) \\ ** Division of Basic Medical Sciences, Faculty of Medicine, Memorial \\ University of Newfoundland, St. John's, Newfoundland, Canada A1B \\ 3V6 (e-mail: vanvliet@mun.ca)
}

\begin{abstract}
:
Salt-induced hypertension has been widely studied in rats, monkeys, chimpanzees and humans. Until recently, the multiple phases of this blood pressure increase to high salt intake had not been closely studied. This work builds upon a recent study, which developed a grey-box multicomponent model of salt-induced hypertension in the Dahl-S rat. The previous 3-component model has been extended here to include additional model dynamics to improve the model fit and add new important elements to the model response. The model was optimised using numerical techniques with experimental data from 4 different protocols with Dahl-S and hybrid rats. Results show a marked improvement over the previous model and confirm the merit of the 5-component model structure.
\end{abstract}

Keywords: hypertension, mathematical model, salt intake, multiple components, Dahl-S

\section{INTRODUCTION}

Salt-induced hypertension ( $\mathrm{SIH}$ ) is a process whereby a subject develops high blood pressure (BP) due to high salt intake. The BP response to salt has been widely studied in chimpanzees (Denton et al. (1995)), spider monkeys (Srinivasan et al. (1980)), rats (Dahl (1961)) and humans (O'Shaughnessy and Karet (2006)).

Until recently, salt-induced hypertension was viewed as a single event and different time courses of the development of hypertension were not appropriately distinguished. This was mainly due to a lack of long term data sampled at short enough intervals to determine different phases of the response. VanVliet et al. (2006) first emphasised the presence of two distinct phases of SIH in Dahl salt-sensitive (Dahl-S) rats. Their study showed that similar effects were also present to various extents in hybrid rats.

Based on this idea, and using the same experimental data, a multi-component grey-box model was created to simulate the acute and progressive time-courses in BP increase due to high salt intake (McLoone et al. (2009)). The model comprises three dynamic components, as shown in Fig. 1, and was optimised for both salt-sensitive and hybrid rats. The model was shown to fit the data reasonably well and possible physiological explanations were assigned to each component. A major drawback, however, is the inability of this 3-component model to account for certain features present in the experimental data.

^ This work was supported by a grant from the Canadian Institutes of Health Research (\#91547), the Ireland-Newfoundland Partnership Fund, the Irish Research Council for Science Engineering and Technology and John and Pat Hume Scholarship fund in NUI Maynooth.
In the present study, the existing 3-component model is extended to improve the data fit and assign a model structure more representative of the underlying physiological system. Two new model components were developed using the average of each experimental protocol (i.e. the 'mean animal'), as it is relatively noise-free data, which emphasises features present in most if not all animals within a data set. Subsequently, the models were parameterised for each individual animal.

The remainder of the paper is organised as follows: Section 2 gives a brief overview of the data sets used in this study and Section 3 shows the model structure development. Section 4 describes the numerical techniques employed for model parameter optimisation, while Sections 5 and 6 present the results and discussion of the work.

\section{EXPERIMENTAL DATA}

Data from four different experimental protocols was used in this study. Plots of the 'mean animal' data for each protocol as well as the respective salt inputs are shown in Figures 2 and 3.

\subsection{Protocol 1}

Experimental protocol 1, described in detail in VanVliet et al. (2006), was conducted on nine 3-month old male Dahl-S rats from the Brookhaven strain. A step increase in dietary salt intake was applied for 6 weeks $(4 \% \mathrm{NaCl})$, followed by a 4-week recovery period with normal salt intake $(0.7 \% \mathrm{NaCl})$. Blood pressure was recorded using telemeters at a rate of a sample per minute. The 24-hour average mean arterial pressure (MAP) was used in the 


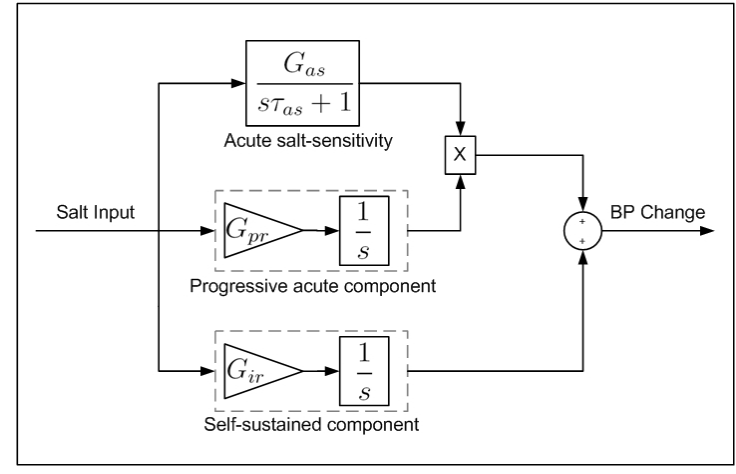

Fig. 1. 3-component model structure

study. Seven Dahl salt-resistant (Dahl-R) rats were used as control subjects and were fed the same diet as the Dahl$S$ rats.
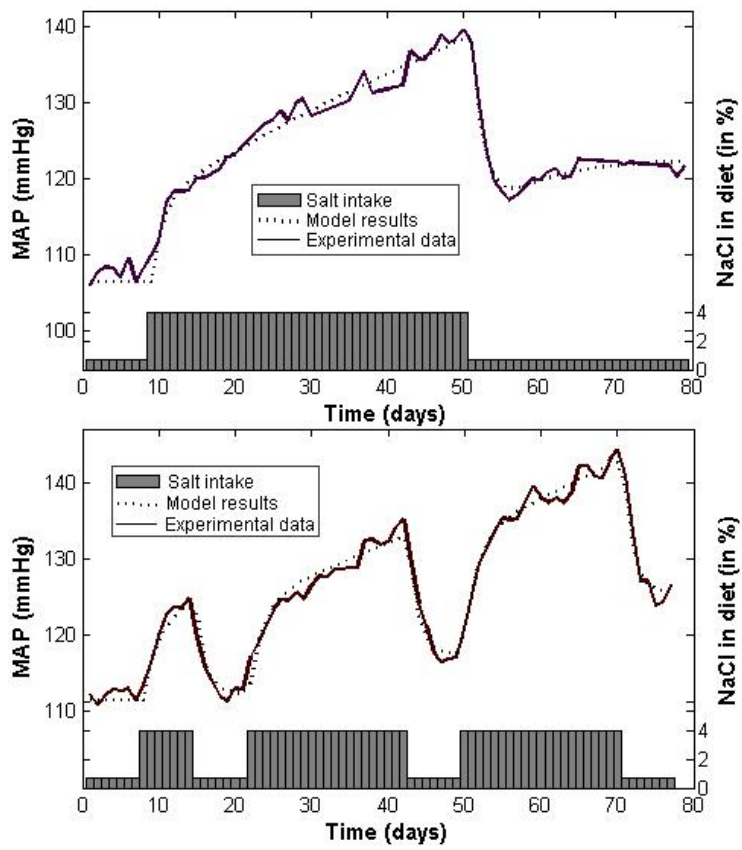

Fig. 2. 5-component model results for the 'mean animal' of Protocols 1 and 2, compared to the experimental data

\subsection{Protocol 2}

Protocol 2 was designed to investigate the reversibility of salt-induced hypertension and was reported in VanVliet et al. (2006). The protocol included variations in salt intake during a course of eleven weeks. The subjects were five male Dahl-S rats, instrumented with telemeters for blood pressure recording. Weeks $1,3,7$ and 11 included normal salt diet of $0.7 \% \mathrm{NaCl}$, while during weeks 2 , 4-6 and 8-10 the animals were given high salt diet of $4 \% \mathrm{NaCl}$.

\subsection{Protocol 3}

Protocol 3, described in McLoone et al. (2009), investigates the effect of short-term changes in salt intake of male Dahl-S rats on their daily mean BP level. The salt levels in the diet were varied in a pseudo-random binary sequence where, at each day of the experiment, the level of salt was either changed or it stayed the same. BP telemeters were implanted in all rats and a recovery period was allowed before a high salt diet was given. The protocol was 72 days long and the daily mean BP level was calculated as the average level of MAP sampled once each minute. The dietary salt level was manipulated in the following manner, 0000000001111110101011001101110110100100 11100010111100101000110000100000 , with each '0' representing a $24 \mathrm{~h}$ exposure to a regular salt diet $(0.7 \% \mathrm{NaCl})$ and each ' 1 ' indicating a $24 \mathrm{~h}$ exposure to high salt diet $(4 \% \mathrm{NaCl})$.

\subsection{Protocol 4}

Protocol 4, similarly to Protocol 1, was conducted to study the BP response to a step increase in salt level in the diet of rats. 13 male FF2-hybrid rats (progeny of the cross between Dahl-S and Dahl-R rats) were studied. Following a control week on a regular salt diet containing $0.7 \% \mathrm{NaCl}$, a high salt diet of $4 \% \mathrm{NaCl}$ was given for 10 weeks and then returned back to a regular salt diet for 1 week. BP was measured using implanted telemeters. The protocol is described in detail in VanVliet et al. (2006).

\section{MODEL DEVELOPMENT}

The existing 3-component model in McLoone et al. (2009) has salt intake levels as input and $\mathrm{BP}$ variation from baseline as output, and consists of the following dynamic components:

- an 'acute salt-sensitivity' component, comprising a first order transfer function with steady state gain $G_{a s}(\mathrm{mmHg} / \% \mathrm{NaCl})$ and time constant $\tau_{a s}$ (days). This component represents the reversible change of BP over a few days to a week.

- a 'progressive self-sustaining' component consisting of a simple integrator with gain $G_{i r}$, and simulating a slow, progressive and irreversible BP increase.

- a 'progressive-acute' component consisting of an integrator term with gain $G_{p r}$, which creates a progressive amplification of the gain $G_{a s}$ of the 'acute salt-sensitivity' component of the model.

\subsection{4 -component model}

As some features of the data were not well represented by the 3-component model in McLoone et al. (2009), expansion of the model was required. In Fig. 4, showing model responses for an animal from Protocol 1, after the high salt stimulus is removed BP recedes to lower levels, undershooting before settling to its new steady-state value. It is impossible to achieve the undershooting response without the addition of an extra component to the existing model.

The fourth, 'compensatory' component, designed to aid model the overshoot in the data, is shown in Fig. 5. It consists of a first order dynamic, whose response opposes the increase in BP due to high salt intake. This is generally consistent with the physiology of the blood pressure control system, which is a complex mixture of balancing neural, hormonal and autoregulatory interactions, and thus it 

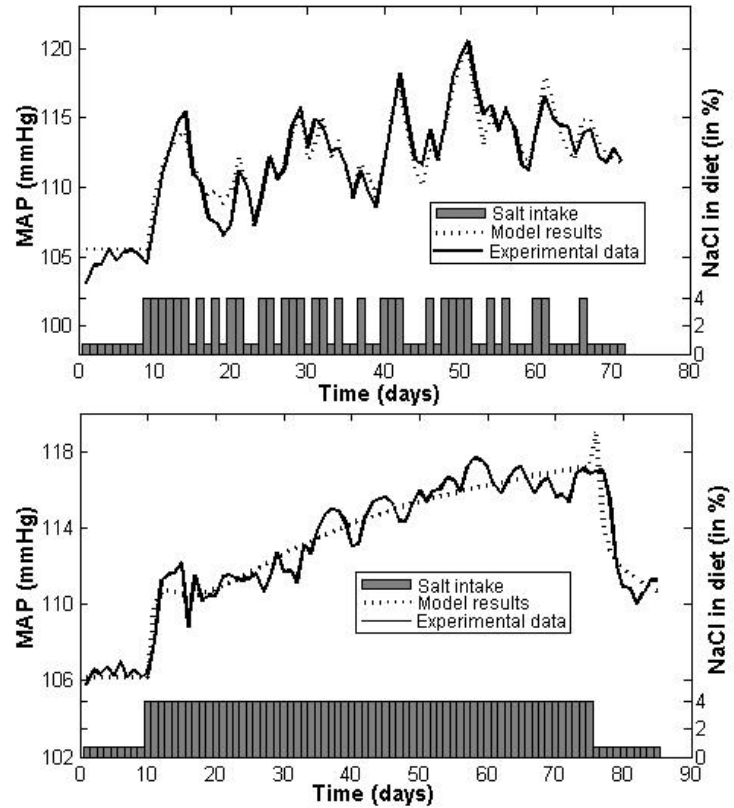

Fig. 3. 5-component model results for the 'mean animal' of Protocols 3 and 4, compared to the experimental data

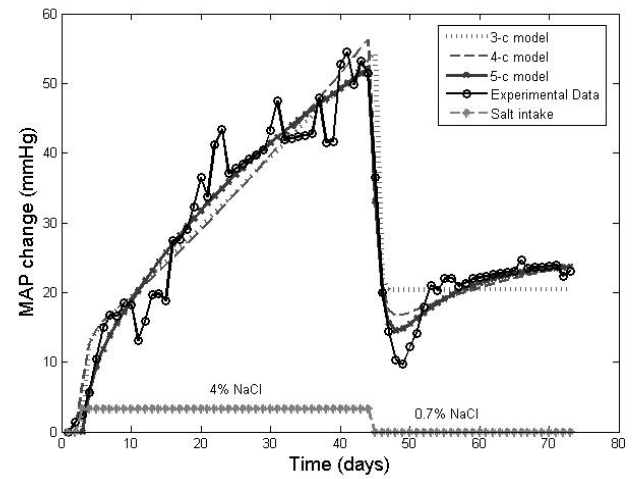

Fig. 4. Model response for all three grey-box models compared to the experimental data for animal SB11

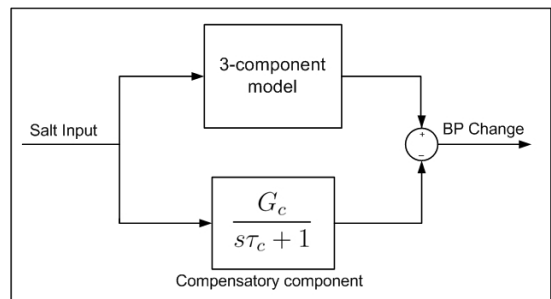

Fig. 5. 4-component model structure

is expected that certain mechanisms will counteract the effect of salt on BP, e.g suppression of angiotensin and aldosterone production (Cholewa and Mattson (2001)).

\subsection{5-component model}

A further set of dynamic components is subsequently added to enhance the model response by accounting for different rates of BP increase and decrease during the

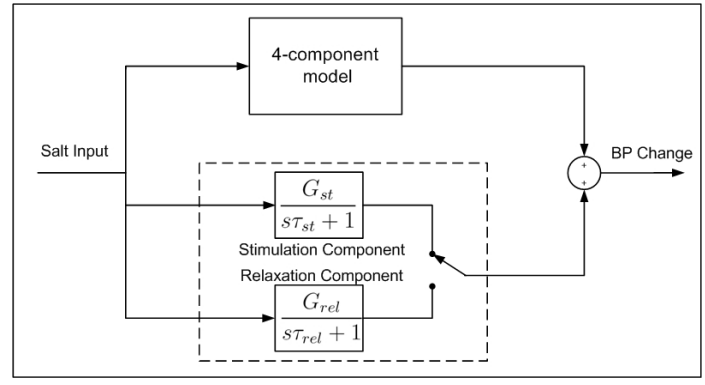

Fig. 6. 5-component model structure

stimulation and relaxation phases of the experiments (increase and decrease of salt contents in the diet). The new model component consists of a switch, along with separate 'stimulation' and 'relaxation' dynamics (Fig. 6). These dynamics work in conjunction with the 'acute saltsensitivity' component, where during high salt intake, the 'stimulation' component is activated, while during normal salt intake, the 'relaxation' component takes over. This is reasonable, since substances involved in BP control may have different formation and dispersion mechanisms and associated activation rates.

\subsection{Neural network model}

Neural networks are black-box models, with potentially unlimited level of complexity and capabilities to model non-linear systems. For comparison purposes, a neural network model was implemented in order to assess whether a purely black-box model would perform better for the existing data sets than the 4- and 5- component models. This would give an estimate of the degree to which the grey-box model assimilates all the dynamics and characteristics contained in the data.

In order to make the models comparable, the output of the neural network is a multi-step ahead prediction. The network was trained separately for each individual animal. As the data sets are very short and all samples were used during training, no validation data was utilised and thus training was stopped after no more than 30 iterations. A small number of neurons was employed to enhance parsimony.

Three single layer and one two-layer dynamic network structures were studied. The three single layer structures had inputs as follows:

- NNET1: the present value of the salt intake and two past values of the BP output,

- NNET2: the present value of the salt intake and three past values of the BP output, and

- NNET3: the present and one past value of the salt intake and two past values of the BP output.

The two-layered neural network (NNET4) had the present value of the salt intake and two past values of the BP output as inputs to the network. All outputs were those predicted by the network. As example, Fig. 7 shows a diagram of NNET1/NNET4. 


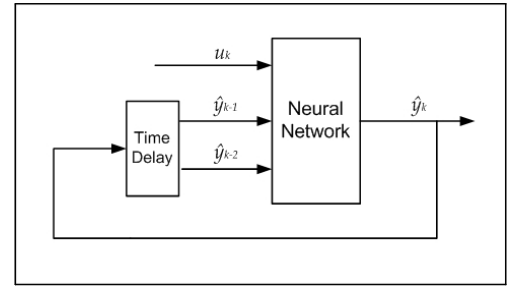

Fig. 7. Example neural network structure, with $u_{k}$ and $\hat{y}_{k}$ being the current salt input and MAP output of the network respectively, and $\hat{y}_{k-1}$ and $\hat{y}_{k-2}$ being the delay input line formed by two past estimated MAP outputs of the network.

\section{MODEL OPTIMISATION}

All grey-box models were parameterised for each individual animal in all 4 protocols and errors were calculated. MatLab's Simulink was used for model implementation, while the parameter optimisation for the grey-box models was done using Nelder-Mead simplex algorithm (Lagarias et al. (1998)). The objective function to be minimised $(J)$ was defined as the Mean Squared Error (MSE) between model results and experimental data:

$$
J=\frac{1}{N} \sum_{i=1}^{N}\left(y_{i}-\hat{y}_{i}\right)^{2}
$$

where,

- $N$ is the number of samples in the data set,

- $\hat{y}_{i}$ is the blood pressure data obtained from the model simulations, and

- $y_{i}$ is the experimental blood pressure data.

The neural network model was implemented using MatLab's Neural Network Toolbox. The nonlinear functions in the hidden layer were logsig, while those in the output layer were linear. The Levenberg-Marquardt algorithm was used for network training. The objective function to be minimised was again the MSE shown above.

\section{RESULTS}

The model results for the 4-component, 5-component and neural network models are presented here, with the results of the 3-component model given for reference.

\subsection{4-component model}

Table 1 lists the mean model parameter values over each protocol for the 4-component model. The 'acute saltsensitivity' gain $G_{a s}$ is clearly lowest for Protocol 4, which is expected as the hybrid animals have a selection of genetic types, varying from salt-sensitive to salt-resistant, while the time constant $\tau_{a s}$ is similar across all protocols. The 'self sustained component' gain $G_{i r}$ is also lowest for the hybrid rats, and highest for the animals of Protocol 1 , as they are exposed to prolonged, uninterrupted high salt intake.

\subsection{5-component model}

Table 2 shows the mean parameter values over each of the four protocols for the 5-component model. As before, the
Table 1. 4-component model mean parameters for Dahl-S rats for all 4 protocols

\begin{tabular}{|c|c|c|c|c|}
\hline Protocol & Protocol1 & Protocol2 & Protocol3 & Protocol4 \\
\hline$G_{a s}$ & 5.792 & 3.819 & 3.076 & 3.466 \\
\hline$\tau_{a s}$ & 2.645 & 2.610 & 2.739 & 3.009 \\
\hline$G_{p r}$ & 0.005 & 0.009 & 0.017 & 0.002 \\
\hline$G_{i r}$ & 0.130 & 0.138 & 0.074 & 0.031 \\
\hline$G_{c}$ & 4.584 & 8.540 & 4.732 & 2.797 \\
\hline$\tau_{c}$ & 18.609 & 30.659 & 19.324 & 39.130 \\
\hline
\end{tabular}

gains of the Protocol 4 animals are consistently lower than those of the salt-sensitive rats.

Table 2. 5-component model mean parameters for Dahl-S rats for all 4 protocols

\begin{tabular}{|c|c|c|c|c|}
\hline Protocol & Protocol1 & Protocol2 & Protocol3 & Protocol4 \\
\hline$G_{a s}$ & 4.785 & 2.748 & 4.017 & 1.841 \\
\hline$\tau_{a s}$ & 3.056 & 1.884 & 2.186 & 1.587 \\
\hline$G_{p r}$ & 0.001 & 0.011 & 0.001 & 0.001 \\
\hline$G_{i r}$ & 0.122 & 0.097 & 0.072 & 0.027 \\
\hline$G_{c}$ & 5.029 & 3.903 & 4.356 & 1.653 \\
\hline$\tau_{c}$ & 9.103 & 13.326 & 7.047 & 5.552 \\
\hline$G_{s t}$ & 4.178 & 2.692 & 3.121 & 1.274 \\
\hline$\tau_{s t}$ & 9.246 & 9.640 & 19.201 & 11.257 \\
\hline$G_{r e l}$ & 3.871 & 1.468 & 0.973 & 2.177 \\
\hline$\tau_{r e l}$ & 3.468 & 3.321 & 0.635 & 1.785 \\
\hline
\end{tabular}

The advantages of the additional 'stimulation' and 'relaxation' components become clear here. The time constant values of the stimulation dynamics $\tau_{s t}$ are prominently larger than those of the relaxation component $\tau_{\text {rel }}$ for all protocols. This helps improve the 5 -component model fit, as shown in Fig. 4, where the responses of the different models are compared for one particular animal (SB11).

A comparison of the mean MSEs and standard deviations (STD) for each protocol is shown in Table 3. Each additional component included in the model leads to a marked reduction in MSE for all 4 protocols.

Table 3. Mean mean squared errors and standard deviations for each model and protocol

\begin{tabular}{|c|c|c|c|}
\hline Protocol1 & 3-comp & 4-comp & 5-comp \\
\hline MSE & 6.664 & 5.362 & 4.066 \\
\hline STD & 5.780 & 3.290 & 1.831 \\
\hline Protocol2 & 3 -comp & 4 -comp & 5 -comp \\
\hline MSE & 9.119 & 7.743 & 6.863 \\
\hline STD & 6.126 & 6.036 & 5.439 \\
\hline Protocol3 & 3 -comp & 4 -comp & 5 -comp \\
\hline MSE & 6.455 & 4.281 & 3.181 \\
\hline STD & 1.950 & 1.410 & 1.115 \\
\hline Protocol4 & 3 -comp & 4 -comp & 5 -comp \\
\hline MSE & 2.935 & 2.578 & 2.261 \\
\hline STD & 3.160 & 2.181 & 1.634 \\
\hline
\end{tabular}

An F-test (Ludden et al. (1994)) was performed to assess whether the increase in the model complexity is justified. In general, an F-test value is calculated to compare the performances of two models, a simple and a more complex one, with respect to the number of their parameters. As the result of the test is a ratio of performances versus complexity between the simpler and the more complex models, an F-test value of 1 signifies that the two models perform equally well for their respective order of complexity, while an F-test value greater than one signifies a merit 
in the use of the more complex model. The degree to which the F-test value exceeds 1 is also a measure of confidence interval on the $\mathrm{F}$-value, depending also on the number of samples in the data set and the number of parameters used. In our study, two F-test values were calculated, one comparing the 3 - and 4-component models (F1) and the other comparing the 4- and 5-component models (F2). The two mean F-test values with their associated confidence intervals are presented in Table 4 for each protocol. From the results it can be seen that increase in complexity both from 3 to 4 components and from 4 to 5 components is justifiable with very high confidence for all protocols except for the one with hybrid animals (Protocol 4). In the hybrid animal data set, the F-test values are greater than 1 ; however the confidence intervals are lower than those for Protocols 1-3. Since some of the hybrid animals have genetic make-up of salt-resistant animals, and thus lack some of the response features of the salt-sensitive animals, it appears that not all model components are necessary to represent their response. It is the intent, however, to have a general model, which can represent both salt-sensitive and salt-resistant animals, thus some model components may inadvertently appear redundant in the latter case but are essential in the former.

Table 4. Mean F-test values for each protocol and associated confidence intervals (P-values). $\mathrm{F} 1$ is the $\mathrm{F}$-value corresponding to the increase of model complexity from three to four components, while F2 refers to the F-value corresponding to the increase of model complexity from four to five components.

\begin{tabular}{|c|c|c|c|c|}
\hline Protocol & F1 & P-value1 & F2 & P-value2 \\
\hline Protocol1 & 5.016 & 0.01 & 4.443 & 0.005 \\
\hline Protocol2 & 10.764 & 0.001 & 2.111 & 0.1 \\
\hline Protocol3 & 15.601 & 0.001 & 5.108 & 0.01 \\
\hline Protocol4 & 2.122 & $>0.1$ & 1.755 & $>0.1$ \\
\hline
\end{tabular}

Another measure of comparison between the models is the residuals test. A plot of the residuals for one animal (SB11) is shown in Fig. 8, while the autocorrelation of the residuals is shown in Fig. 9. Both plots confirm improvement with the addition of component 4 and then component 5 to the model. With each new component the residuals become less correlated, thus approaching white noise. This also shows that the majority of the signal is explained by the 5-component model and the addition of any further component would not be required, as the small improvement in fit would be unlikely to justify the increase in complexity. However, the examination of an unstructured black-box model may reveal the degree to which outstanding correlated data can be modelled.

Figures 2 and 3 show plots of the 5-component model results for the 'mean animal' for each protocol and compare them to the experimental data. These plots confirm the quality of the model fit and give merit to the model structure.

\subsection{Neural networks}

Table 5 shows the mean MSEs and standard deviations for the various neural network models. Surprisingly, results are not as good as those for the grey-box models. There

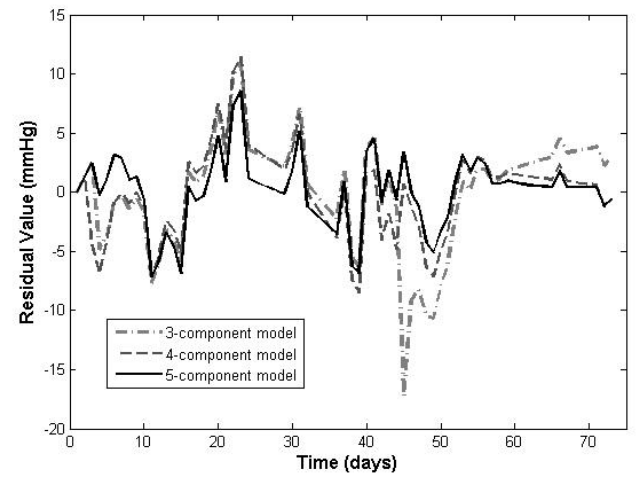

Fig. 8. Residuals for one animal from Protocol 1 (SB11)

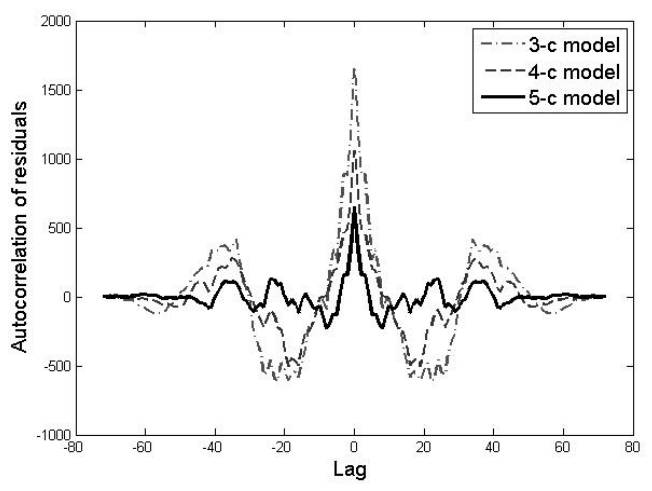

Fig. 9. Autocorrelation of residuals for one animal from Protocol 1 (SB11)

are a number of potential reasons for the poor response of the neural networks. The data sets have very few samples $(<80)$ and the networks were trained on individual animals only. No validation data was used, therefore training had to be stopped early to avoid over-fitting. With further refinement of the neural network models arguably better results could have been obtained. However, the purpose was to assess how well models with strictly defined structure and based on some physiological principles (the 4- and 5-component models) could perform compared to purely black box models.

Table 5. Mean MSEs and STDs for the neural network models for all protocols

\begin{tabular}{|c|c|c|c|c|}
\hline Protocol1 & NNET1 & NNET2 & NNET3 & NNET4 \\
\hline$M S E$ & 11.860 & 16.754 & 12.960 & 19.461 \\
\hline STD & 8.699 & 12.142 & 10.469 & 23.965 \\
\hline Protocol2 & NNET1 & NNET2 & NNET3 & NNET4 \\
\hline$M S E$ & 27.788 & 32.044 & 47.550 & 22.833 \\
\hline STD & 23.892 & 23.070 & 45.332 & 14.992 \\
\hline Protocol3 & NNET1 & NNET2 & NNET3 & NNET4 \\
\hline$M S E$ & 11.586 & 14.278 & 15.073 & 12.168 \\
\hline STD & 3.335 & 5.783 & 6.528 & 4.968 \\
\hline Protocol4 & NNET1 & NNET2 & NNET3 & NNET4 \\
\hline$M S E$ & 5.241 & 6.007 & 4.411 & 4.957 \\
\hline STD & 4.711 & 4.860 & 2.633 & 3.184 \\
\hline
\end{tabular}

\section{DISCUSSION}

This study highlights the multi-component nature of saltinduced hypertension by describing two additional model 
components, of different complexity, which represent the time course of BP response to high salt intake in Dahl-S and hybrid rats. Not all features of the BP response to high salt intake are evident in every protocol due to the various salt intake sequences used as excitation, therefore the 'mean animal' of all 4 protocols was used in the model development stage in order to obtain a generic structure with the ability to represent a variety of responses. The previous 3-component model by McLoone et al. (2009) represented a good starting point in the modelling of saltinduced hypertension but lacked the complexity to depict all major features of the $\mathrm{BP}$ response. Table 3 shows the large reduction in error in the 5-component model compared to the initial 3-component model.

The 5-component model developed here is so far the most comprehensive model of salt-induced hypertension in Dahl-S rats. The model fits the data very well and the components are reasonably intuitive and have some physiological basis. The autocorrelation of the residuals approaches that of white noise, justifying the completeness of the model structure.

Fig. 4 also shows the type of improvement in model response that the additional components (4 and 5) bring to the model over the 3-component model. New elements of the data are featured, in particular the differing time constants between stimulation and relaxation and the undershoot of BP when high-salt intake is ceased.

The neural network models, and their inferior performance compared to the grey-box models, have manifested the importance in using physiological knowledge in model development. Arguably, the neural network models could have been further refined in order to achieve better MSE results, however it is futile to strive for minimal errors, when no practical knowledge can be gained from the system. Moreover, this one-to-one comparison emphasises again that any further increase in model complexity would not necessarily improve the 5-component grey-box model response.

The 5-component model has helped gain an insight into the processes involved in blood pressure control and saltinduced hypertension and it is important for future experimental protocols to be designed in view of the fact the $\mathrm{BP}$ increase due to salt intake has numerous components, some reversible and others irreversible. It is not within our expertise to assign exact physiological meaning to the various additional dynamics in the model. Nevertheless, it is believed that the 5-component model articulates clear and distinct characteristics which, we believe, are caused by separate physiological phenomena. The combination of 'acute salt-sensitivity' and 'progressive acute' components coincides with the time course of re-establishment of salt balance within days. The 'self-sustained' component, leading to an irreversibility of BP increase, could be attributed to the vascular remodelling and/or renal damage such as fibrosis and hypertrophy. The 'compensatory' component, could be related to mechanisms such as the suppression of the renin-angiotensin-aldosterone system, which opposes $\mathrm{BP}$ increase, while the combination of 'stimulation' and 'relaxation' dynamics, can be attributed to a hysteresis effect, typical of systems where substances are produced and dispersed at different rates.
We believe that appropriate experiments should be conducted in order to differentiate between the various response components and to attempt to understand their exact physiological significance. Furthermore, we know from Dahl's studies (Dahl (1961); Dahl and Schackow (1964)) that hypertension has genetic etiology and that multiple genes are involved in the $\mathrm{BP}$ increase due to salt, therefore it would be beneficial to utilise the multicomponent model presented here to determine if different genes are responsible for the different response components and also to establish what those genes are.

In the future, additional excitation signals and protocols may be studied in order to provide the model with the ability to model inter-animal variation in BP, as presently the model is optimised to simulate the response of individual animals only. In addition, experiments similar to the ones described in this work, are currently carried out on Sprague Dawley rats in Prof. Van Vliet's Laboratory. These would help confirm the suitability of the 5component model structure to fit data from a wider variety of animals, ranging from normotensive to hypertensive.

\section{REFERENCES}

Cholewa, B. and Mattson, D. (2001). Role of the reninangiotensin system during alterations of sodium intake in conscious mice. Am. J. Physiol. Regul. Integ. Comp. Physiol., 281, R987-R993.

Dahl, L. (1961). Effects of chronic excess salt feeding. $J$. Exp. Med., 114, 231-236.

Dahl, L. and Schackow, E. (1964). Effects of chronic excess salt ingestion: Experimental hypertension in the rat. Can Med Ass J., 90, 155-160.

Denton, D., Weisinger, R., Mundy, N., Wickings, E., Dixons, A., Moisson, P., Pingard, A., Shade, R., Carey, D., Ardaillou, R., Paillard, F., Chapman, J., Thillet, J., and Michel, J. (1995). The effect of increased salt intake on blood pressure of chimpanzees. Nature Medicine, 1 (10), 1009-1016.

Lagarias, J., Reeds, J., Wright, M., and Wright, P. (1998). Convergence properties of the nelder-mead simplex method in low dimensions. SIAM Journal of Optimization, 9 (1), 112-147.

Ludden, T., Beal, S., and Sheiner, L. (1994). Comparison of the akaike information criterion, the schwarz criterion and the $\mathrm{f}$ test as guides to model selection. Journal of Pharmac. and Biopharm., 22 (5), 431-445.

McLoone, V., Ringwood, J., and VanVliet, B. (2009). Multi-component mathematical model of salt sensitivity in dahl-s rats. Submitted to Hypertension.

O'Shaughnessy, K. and Karet, F. (2006). Salt handling and hypertension. Ann. Rev. Nutr., 26, 343-365.

Srinivasan, S., Berenson, G., Radhakrishnamurthy, B., Dalferes, E., Underwood, D., and Foster, T. (1980). Effects of dietary sodium and sucrose on the induction of hypertension in spider monkeys. Am. J. Clin. Nutr., $33,561-569$.

VanVliet, B., Chafe, L., S.Halfyard, and Leonard, A. (2006). Distinct rapid and slow phases of salt-induced hypertension in dahl salt-sensitive rats. J Hypertension, 24. 